\title{
CHANGES IN THE DEPTH OF THE ANTERIOR CHAMBER AND IN THE RADIUS OF CURVATURE OF THE FRONT SURFACE OF THE LENS DURING GROWTH* OBSERVATIONS ON THE RABBIT
}

\author{
BY \\ ARNOLD SORSBY $\dagger$, JANET STONE, G. A. LEARY, AND \\ MICHAEL SHERIDAN \\ Ophthalmological Research Department, Royal College of Surgeons and Royal Eye Hospital
}

IN an earlier study (Sorsby and Sheridan, 1953) attention was drawn to the theoretical need for a considerable reduction in the power of the cornea and of the lens during growth to compensate for axial elongation-seeing that the refraction of the baby's eye is not markedly different from that of the adult, whilst the elongation presupposes a change of some 20-30 D. This theoretical assumption was tested in the rabbit: four animals between the ages of 4 to 30 weeks were followed up, and a marked reduction was registered for the cornea, the power declining from over $60 \mathrm{D}$ to well below $50 \mathrm{D}$ over that period. It was subsequently shown (Sorsby, Benjamin, Davey, Sheridan, and Tanner, 1957) that the corneal power stabilizes at around $43 \mathrm{D}$, by 60 to 80 weeks of age. Similar findings have been recorded by Chou (1954) and by Stone and Leary (1957).

In the present study an account is given of the changes observed in the depth of the anterior chamber and in the curvature of the front surface of the lens in rabbits followed up from 7 to 80 weeks of age. A photographic method, recorded elsewhere (Sorsby, Benjamin, and Sheridan, 1961), was used to obtain these measurements. No data are available on the thickness of the lens, or on the radius of curvature of the back surface of the lens owing to technical difficulties; the rabbit lens is thick and not very suitable for the photographic method which was evolved for use on the human eye with its very much flatter lens. In all, seventeen rabbits were studied and the measurements recorded are shown in the Table (overleaf).

Figs 1 and 2 (overleaf) show the corresponding mean growth curves.

It will be seen that the depth of the anterior chamber increases by $0.6 \mathrm{~mm}$. up to 20 weeks of age and thereafter remains relatively stationary at about $2.9 \mathrm{~mm}$.; it had therefore already reached $4 / 5$ of its final depth at 7 weeks of age. The front surface of the lens continues to flatten at least up to 60 weeks, and possibly up to 80 weeks. It is apparent from Fig. 2 that the rate of flattening slows down after the 12th week: the total amount of flattening recorded is $1.8 \mathrm{~mm}$., half of which occurred in the first 13 weeks of

- Received for publication September 10, 1959.

† Working with a grant from the Alexander Pigott Wernher Memorial Trust, Medical Research Council. 
TABLE

DEPTH OF ANTERIOR CHAMBER AND RADIUS OF CURVATURE OF FRONT SURFACE

\begin{tabular}{|c|c|c|c|c|c|c|c|c|c|c|}
\hline \multirow{3}{*}{$\begin{array}{c}\text { Rabbit } \\
\text { No. }\end{array}$} & \multirow{3}{*}{ Eye } & \multicolumn{9}{|c|}{ Measurement: Depth of Anterior Chamber (mm.) } \\
\hline & & \multicolumn{9}{|c|}{ Age (wks) } \\
\hline & & 7 & 12 & 20 & 30 & 40 & 50 & 60 & 70 & 80 \\
\hline H1 & $\begin{array}{l}\text { R. } \\
\text { L. }\end{array}$ & $\begin{array}{l}2 \cdot 2 \\
2 \cdot 2\end{array}$ & $\begin{array}{l}2 \cdot 8 \\
2 \cdot 9\end{array}$ & $\begin{array}{l}2 \cdot 7 \\
2 \cdot 7\end{array}$ & $\begin{array}{l}3 \cdot 1 \\
3 \cdot 0\end{array}$ & $\begin{array}{l}2.9 \\
2 \cdot 9\end{array}$ & $\begin{array}{l}2.5 \\
2.6\end{array}$ & $\begin{array}{l}2 \cdot 9 \\
2 \cdot 6\end{array}$ & $\begin{array}{l}3 \cdot 2 \\
2 \cdot 9\end{array}$ & $\begin{array}{l}3 \cdot 1 \\
2 \cdot 8\end{array}$ \\
\hline $\mathbf{H} 2$ & $\begin{array}{l}\text { R. } \\
\text { L. }\end{array}$ & $\begin{array}{l}2 \cdot 1 \\
2 \cdot 4\end{array}$ & $\begin{array}{l}3 \cdot 0 \\
3 \cdot 1\end{array}$ & $\begin{array}{l}3 \cdot 1 \\
2 \cdot 6\end{array}$ & $\begin{array}{l}2 \cdot 7 \\
3 \cdot 1\end{array}$ & $\begin{array}{l}2 \cdot 9 \\
2 \cdot 8\end{array}$ & $\begin{array}{l}3 \cdot 0 \\
2 \cdot 8\end{array}$ & $\begin{array}{l}3.4 \\
3.0\end{array}$ & $\begin{array}{l}2 \cdot 7 \\
3 \cdot 2\end{array}$ & $\begin{array}{l}3 \cdot 0 \\
2 \cdot 7\end{array}$ \\
\hline $\mathbf{H 3}$ & $\begin{array}{l}\text { R. } \\
\text { L. }\end{array}$ & $\begin{array}{l}2 \cdot 3 \\
2 \cdot 3\end{array}$ & $\begin{array}{l}2 \cdot 1 \\
2 \cdot 7\end{array}$ & $\begin{array}{l}2.7 \\
3.0\end{array}$ & $\begin{array}{l}3 \cdot 2 \\
3 \cdot 3\end{array}$ & $\begin{array}{l}3 \cdot 1 \\
3 \cdot 1\end{array}$ & $\begin{array}{l}2 \cdot 6 \\
2 \cdot 7\end{array}$ & $\begin{array}{l}3 \cdot 1 \\
2 \cdot 7\end{array}$ & $\begin{array}{l}3 \cdot 1 \\
3 \cdot 0\end{array}$ & $\begin{array}{l}3 \cdot 2 \\
3 \cdot 2\end{array}$ \\
\hline H4 & $\begin{array}{l}\text { R. } \\
\text { L. }\end{array}$ & $\begin{array}{l}2 \cdot 4 \\
2 \cdot 7\end{array}$ & $\begin{array}{l}2 \cdot 9 \\
2 \cdot 8\end{array}$ & $\begin{array}{l}3 \cdot 1 \\
3 \cdot 1\end{array}$ & $\begin{array}{l}3 \cdot 0 \\
3 \cdot 0\end{array}$ & $\begin{array}{l}3 \cdot 1 \\
2 \cdot 6\end{array}$ & $\begin{array}{l}3.0 \\
3.0\end{array}$ & $\begin{array}{l}2 \cdot 7 \\
2 \cdot 4\end{array}$ & $\begin{array}{l}3 \cdot 1 \\
3 \cdot 1\end{array}$ & $\begin{array}{l}2 \cdot 9 \\
3 \cdot 2\end{array}$ \\
\hline H5 & $\begin{array}{l}\text { R. } \\
\text { L. }\end{array}$ & $\begin{array}{l}2 \cdot 6 \\
2 \cdot 5\end{array}$ & $\begin{array}{l}2 \cdot 7 \\
2 \cdot 8\end{array}$ & $\begin{array}{l}3 \cdot 0 \\
2.6\end{array}$ & $\begin{array}{l}3 \cdot 0 \\
3 \cdot 1\end{array}$ & $\begin{array}{l}2.9 \\
3.0\end{array}$ & \multicolumn{4}{|c|}{ Rabbit died } \\
\hline H6 & $\begin{array}{l}\text { R. } \\
\text { L. }\end{array}$ & $\begin{array}{l}2 \cdot 5 \\
2 \cdot 4\end{array}$ & $\begin{array}{l}2 \cdot 6 \\
2 \cdot 6\end{array}$ & $\begin{array}{l}3 \cdot 2 \\
3 \cdot 0\end{array}$ & $\begin{array}{l}3 \cdot 2 \\
3 \cdot 1\end{array}$ & $\begin{array}{l}3 \cdot 2 \\
3 \cdot 1\end{array}$ & $\begin{array}{l}3 \cdot 0 \\
2 \cdot 6\end{array}$ & $\begin{array}{l}2 \cdot 7 \\
2 \cdot 5\end{array}$ & $\begin{array}{l}3 \cdot 3 \\
3 \cdot 4\end{array}$ & \\
\hline G2 & $\begin{array}{l}\text { R. } \\
\text { L. }\end{array}$ & & $\begin{array}{l}2 \cdot 7 \\
3 \cdot 5\end{array}$ & $\begin{array}{l}2.6 \\
2.8\end{array}$ & $\begin{array}{l}2 \cdot 6 \\
2 \cdot 7\end{array}$ & $\begin{array}{l}2 \cdot 7 \\
2 \cdot 7\end{array}$ & $\begin{array}{l}2 \cdot 9 \\
3 \cdot 3\end{array}$ & $\begin{array}{l}3 \cdot 0 \\
2 \cdot 7\end{array}$ & $\begin{array}{l}2.9 \\
2 \cdot 7\end{array}$ & \\
\hline G3 & $\begin{array}{l}\text { R. } \\
\text { L. }\end{array}$ & & $\begin{array}{r}2 \cdot 8 \\
3.0\end{array}$ & $\begin{array}{l}3 \cdot 3 \\
3 \cdot 3\end{array}$ & $\begin{array}{l}2 \cdot 7 \\
2 \cdot 5\end{array}$ & $\begin{array}{l}3 \cdot 1 \\
2 \cdot 8\end{array}$ & $\begin{array}{l}3 \cdot 3 \\
3.0\end{array}$ & & $\begin{array}{l}2 \cdot 9 \\
2 \cdot 5\end{array}$ & \\
\hline G4 & $\begin{array}{l}\text { R. } \\
\text { L. }\end{array}$ & & $\begin{array}{l}2 \cdot 9 \\
2 \cdot 6\end{array}$ & $\begin{array}{l}2 \cdot 8 \\
2 \cdot 9\end{array}$ & $\begin{array}{l}2 \cdot 6 \\
2 \cdot 9\end{array}$ & $\begin{array}{l}2 \cdot 8 \\
3 \cdot 1\end{array}$ & $\begin{array}{l}3.0 \\
2.9\end{array}$ & $\begin{array}{l}2 \cdot 8 \\
2 \cdot 7\end{array}$ & $\begin{array}{l}3 \cdot 1 \\
2 \cdot 7\end{array}$ & $\begin{array}{l}2.5 \\
3.0\end{array}$ \\
\hline G5 & $\begin{array}{l}\text { R. } \\
\text { L. }\end{array}$ & & $\begin{array}{l}2 \cdot 5 \\
2 \cdot 6\end{array}$ & $\begin{array}{l}3 \cdot 2 \\
3 \cdot 1\end{array}$ & $\begin{array}{l}2 \cdot 8 \\
2 \cdot 7\end{array}$ & $\begin{array}{l}3 \cdot 0 \\
2 \cdot 9\end{array}$ & $\begin{array}{l}2.9 \\
2 \cdot 9\end{array}$ & $\begin{array}{l}2.9 \\
2.9\end{array}$ & $\begin{array}{l}3 \cdot 3 \\
2 \cdot 7\end{array}$ & $\begin{array}{l}2 \cdot 9 \\
2 \cdot 7\end{array}$ \\
\hline F3 & $\begin{array}{l}\text { R. } \\
\text { L. }\end{array}$ & & $\begin{array}{l}2 \cdot 9 \\
2 \cdot 6\end{array}$ & $\begin{array}{l}3.0 \\
3.0\end{array}$ & $\begin{array}{l}3 \cdot 3 \\
3 \cdot 6\end{array}$ & $\begin{array}{l}2 \cdot 9 \\
3 \cdot 1\end{array}$ & $\begin{array}{l}3.0 \\
2.9\end{array}$ & $\begin{array}{l}3.0 \\
3.0\end{array}$ & $\begin{array}{l}3 \cdot 0 \\
3 \cdot 0\end{array}$ & $\begin{array}{l}2 \cdot 9 \\
2 \cdot 8\end{array}$ \\
\hline F6 & $\begin{array}{l}\text { R. } \\
\text { L. }\end{array}$ & & $\begin{array}{l}2 \cdot 7 \\
2 \cdot 8\end{array}$ & $\begin{array}{l}2 \cdot 9 \\
2 \cdot 3\end{array}$ & $\begin{array}{l}2 \cdot 6 \\
2 \cdot 9\end{array}$ & $\begin{array}{l}2 \cdot 5 \\
3 \cdot 1\end{array}$ & $\begin{array}{l}3.0 \\
2 \cdot 9\end{array}$ & & $\begin{array}{l}2.9 \\
3.0\end{array}$ & $\begin{array}{l}3 \cdot 2 \\
2 \cdot 8\end{array}$ \\
\hline F8 & $\begin{array}{l}\text { R. } \\
\text { L. }\end{array}$ & & $\begin{array}{l}2 \cdot 5 \\
2 \cdot 7 \\
\end{array}$ & $\begin{array}{r}2 \cdot 9 \\
3 \cdot 2 \\
\end{array}$ & $\begin{array}{l}3 \cdot 1 \\
2 \cdot 9\end{array}$ & $\begin{array}{l}3 \cdot 0 \\
3 \cdot 2\end{array}$ & $\begin{array}{l}3 \cdot 1 \\
2 \cdot 8\end{array}$ & $\begin{array}{l}2 \cdot 9 \\
2 \cdot 6\end{array}$ & $\begin{array}{l}2 \cdot 6 \\
2 \cdot 8\end{array}$ & \\
\hline E1 & $\begin{array}{l}\text { R. } \\
\text { L. }\end{array}$ & & & & $\begin{array}{l}2 \cdot 6 \\
2 \cdot 9\end{array}$ & $\begin{array}{l}2 \cdot 8 \\
2 \cdot 8\end{array}$ & $\begin{array}{l}2.9 \\
2 \cdot 7\end{array}$ & $\begin{array}{l}2 \cdot 8 \\
3: 1\end{array}$ & $\begin{array}{l}2 \cdot 5 \\
2 \cdot 8\end{array}$ & $\begin{array}{l}2.7 \\
2.9\end{array}$ \\
\hline E2 & $\begin{array}{l}\text { R. } \\
\text { L. }\end{array}$ & & & & $\begin{array}{l}2 \cdot 6 \\
3 \cdot 0\end{array}$ & $\begin{array}{l}3 \cdot 2 \\
3 \cdot 3\end{array}$ & $\begin{array}{l}2 \cdot 8 \\
2 \cdot 7\end{array}$ & $\begin{array}{l}3 \cdot 7 \\
2 \cdot 7\end{array}$ & $\begin{array}{l}2 \cdot 8 \\
2 \cdot 6\end{array}$ & $\begin{array}{l}3 \cdot 0 \\
2 \cdot 8\end{array}$ \\
\hline $\mathrm{Cl}$ & $\begin{array}{l}\text { R. } \\
\text { L. }\end{array}$ & & & $\begin{array}{l}3.4 \\
3.5 \\
\end{array}$ & $\begin{array}{l}3 \cdot 0 \\
3 \cdot 2 \\
\end{array}$ & $\begin{array}{l}2 \cdot 6 \\
3 \cdot 5\end{array}$ & $\begin{array}{l}3 \cdot 1 \\
3 \cdot 1\end{array}$ & $\begin{array}{l}3 \cdot 2 \\
2 \cdot 8\end{array}$ & $\begin{array}{l}3.0 \\
2.9\end{array}$ & \\
\hline $\mathrm{C2}$ & $\begin{array}{l}\text { R. } \\
\text { L. }\end{array}$ & & & & $\begin{array}{l}3 \cdot 0 \\
3 \cdot 1\end{array}$ & $\begin{array}{l}2.9 \\
2.9\end{array}$ & $\begin{array}{l}3 \cdot 5 \\
2 \cdot 8\end{array}$ & $\begin{array}{l}3 \cdot 0 \\
3 \cdot 1\end{array}$ & $\begin{array}{l}2 \cdot 8 \\
3 \cdot 0\end{array}$ & $\begin{array}{l}2 \cdot 7 \\
3 \cdot 2\end{array}$ \\
\hline $\begin{array}{l}\text { Mear } \\
\text { Stan } \\
\text { Devi }\end{array}$ & & $\begin{array}{c}2 \cdot 4 \\
\pm 0 \cdot 17\end{array}$ & $\begin{array}{c}2 \cdot 8 \\
\pm 0 \cdot 25\end{array}$ & $\begin{array}{c}3 \cdot 0 \\
\pm 0 \cdot 27\end{array}$ & $\begin{array}{c}2.9 \\
\pm 0 \cdot 25\end{array}$ & $\begin{array}{c}3.0 \\
\pm 0.21\end{array}$ & $\begin{array}{c}2 \cdot 9 \\
\pm 0 \cdot 21\end{array}$ & $\begin{array}{c}2.9 \\
\pm 0.27\end{array}$ & $\begin{array}{c}2 \cdot 9 \\
\pm 0.23\end{array}$ & $\begin{array}{c}2.9 \\
\pm 0.20\end{array}$ \\
\hline
\end{tabular}


JF LENS IN SEVENTEEN RABBITS FOLLOWED UP FROM 7 TO 80 WEEKS OF AGE

\begin{tabular}{|c|c|c|c|c|c|c|c|c|c|c|}
\hline \multicolumn{9}{|c|}{ Measurement: Radius of Front Surface of Lens (mm.) } & \multirow{3}{*}{ Eye } & \multirow{3}{*}{$\begin{array}{l}\text { Rabbit } \\
\text { No. }\end{array}$} \\
\hline \multicolumn{9}{|c|}{ Age (wks) } & & \\
\hline 7 & 12 & 20 & 30 & 40 & 50 & 60 & 70 & 80 & & \\
\hline $\begin{array}{l}5 \cdot 6 \\
5 \cdot 4\end{array}$ & $\begin{array}{l}5 \cdot 7 \\
5 \cdot 7\end{array}$ & $\begin{array}{l}6 \cdot 2 \\
6 \cdot 6\end{array}$ & $\begin{array}{l}6 \cdot 6 \\
6 \cdot 4\end{array}$ & $\begin{array}{l}6 \cdot 6 \\
6 \cdot 7\end{array}$ & $\begin{array}{l}7 \cdot 3 \\
7 \cdot 0\end{array}$ & $\begin{array}{l}7 \cdot 2 \\
7 \cdot 2\end{array}$ & $\begin{array}{l}7 \cdot 0 \\
6.6\end{array}$ & $\begin{array}{l}7 \cdot 0 \\
7 \cdot 2\end{array}$ & $\begin{array}{l}\text { R. } \\
\text { L. }\end{array}$ & H1 \\
\hline $\begin{array}{l}5 \cdot 6 \\
5 \cdot 1\end{array}$ & $\begin{array}{l}5 \cdot 2 \\
5 \cdot 5\end{array}$ & $\begin{array}{l}5.9 \\
6 \cdot 2\end{array}$ & $\begin{array}{l}6 \cdot 1 \\
6 \cdot 2\end{array}$ & $\begin{array}{l}6 \cdot 8 \\
7 \cdot 3\end{array}$ & $\begin{array}{l}6 \cdot 8 \\
7 \cdot 0\end{array}$ & $\begin{array}{l}6.9 \\
6.9\end{array}$ & $\begin{array}{l}8 \cdot 1 \\
7 \cdot 3\end{array}$ & $\begin{array}{l}7 \cdot 0 \\
7 \cdot 7\end{array}$ & $\begin{array}{l}\text { R. } \\
\text { L. }\end{array}$ & H2 \\
\hline $\begin{array}{l}5 \cdot 6 \\
5 \cdot 3\end{array}$ & $\begin{array}{l}6 \cdot 1 \\
5 \cdot 7\end{array}$ & $\begin{array}{l}6.0 \\
5.8\end{array}$ & $\begin{array}{l}7 \cdot 4 \\
6 \cdot 8\end{array}$ & $\begin{array}{l}6 \cdot 8 \\
6 \cdot 8\end{array}$ & $\begin{array}{l}7 \cdot 1 \\
7 \cdot 6\end{array}$ & $\begin{array}{l}7 \cdot 3 \\
7 \cdot 1\end{array}$ & $\begin{array}{l}6 \cdot 7 \\
6 \cdot 2\end{array}$ & $\begin{array}{l}7 \cdot 2 \\
6 \cdot 5\end{array}$ & $\begin{array}{l}\text { R. } \\
\text { L. }\end{array}$ & H3 \\
\hline $\begin{array}{l}4 \cdot 8 \\
5 \cdot 1\end{array}$ & $\begin{array}{l}5 \cdot 6 \\
6 \cdot 2\end{array}$ & $\begin{array}{l}6 \cdot 1 \\
6 \cdot 2\end{array}$ & $\begin{array}{l}6 \cdot 4 \\
7 \cdot 3\end{array}$ & $\begin{array}{l}6 \cdot 5 \\
6 \cdot 3\end{array}$ & $\begin{array}{l}6 \cdot 8 \\
6.9\end{array}$ & $\begin{array}{l}7 \cdot 3 \\
7 \cdot 2\end{array}$ & $\begin{array}{l}6 \cdot 3 \\
7 \cdot 4\end{array}$ & $\begin{array}{l}7 \cdot 8 \\
6 \cdot 8\end{array}$ & $\begin{array}{l}\text { R. } \\
\text { L. }\end{array}$ & $\mathrm{H} 4$ \\
\hline $\begin{array}{l}4 \cdot 7 \\
4 \cdot 2\end{array}$ & $\begin{array}{l}6 \cdot 0 \\
6 \cdot 2\end{array}$ & $\begin{array}{l}6 \cdot 2 \\
6 \cdot 2\end{array}$ & $\begin{array}{l}6 \cdot 3 \\
5 \cdot 7\end{array}$ & $\begin{array}{l}6 \cdot 7 \\
6 \cdot 4\end{array}$ & \multicolumn{4}{|c|}{ Rabbit died } & $\begin{array}{l}\text { R. } \\
\text { L. }\end{array}$ & H5 \\
\hline \multirow[t]{12}{*}{$\begin{array}{l}5.4 \\
5.6\end{array}$} & $\begin{array}{l}6 \cdot 0 \\
6 \cdot 5\end{array}$ & $\begin{array}{l}5.9 \\
6.3\end{array}$ & $\begin{array}{l}6 \cdot 2 \\
6 \cdot 2\end{array}$ & $\begin{array}{l}6 \cdot 8 \\
6 \cdot 7\end{array}$ & $\begin{array}{l}7 \cdot 1 \\
6 \cdot 4\end{array}$ & $\begin{array}{l}7 \cdot 2 \\
6 \cdot 9\end{array}$ & $\begin{array}{l}7.0 \\
6.7\end{array}$ & & $\begin{array}{l}\text { R. } \\
\text { L. }\end{array}$ & H6 \\
\hline & $\begin{array}{l}6 \cdot 5 \\
5 \cdot 8\end{array}$ & $\begin{array}{l}6 \cdot 2 \\
6 \cdot 4\end{array}$ & $\begin{array}{l}7 \cdot 0 \\
6 \cdot 3\end{array}$ & $\begin{array}{l}6 \cdot 5 \\
7 \cdot 2\end{array}$ & $\begin{array}{l}6 \cdot 8 \\
6 \cdot 6\end{array}$ & $\begin{array}{l}6 \cdot 8 \\
7 \cdot 1\end{array}$ & $\begin{array}{l}6.9 \\
7.0\end{array}$ & & $\begin{array}{l}\text { R. } \\
\text { L. }\end{array}$ & G2 \\
\hline & $\begin{array}{l}5 \cdot 5 \\
5 \cdot 8\end{array}$ & $\begin{array}{l}5 \cdot 7 \\
5 \cdot 5\end{array}$ & $\begin{array}{l}6 \cdot 5 \\
6 \cdot 5\end{array}$ & $\begin{array}{l}6 \cdot 2 \\
6 \cdot 5\end{array}$ & $\begin{array}{l}5 \cdot 7 \\
6 \cdot 5\end{array}$ & & $\begin{array}{l}6 \cdot 5 \\
6 \cdot 6\end{array}$ & & $\begin{array}{l}\text { R. } \\
\text { L. }\end{array}$ & G3 \\
\hline & $\begin{array}{l}5 \cdot 4 \\
5 \cdot 6\end{array}$ & $\begin{array}{l}6 \cdot 8 \\
6 \cdot 1\end{array}$ & $\begin{array}{l}6 \cdot 2 \\
6 \cdot 8\end{array}$ & $\begin{array}{l}6 \cdot 1 \\
6 \cdot 5\end{array}$ & $\begin{array}{l}6 \cdot 8 \\
6 \cdot 1\end{array}$ & $\begin{array}{l}6 \cdot 0 \\
6 \cdot 8\end{array}$ & $\begin{array}{l}6 \cdot 9 \\
6 \cdot 6\end{array}$ & $\begin{array}{l}7 \cdot 1 \\
7 \cdot 1\end{array}$ & $\begin{array}{l}\text { R. } \\
\text { L. }\end{array}$ & G4 \\
\hline & $\begin{array}{l}5 \cdot 8 \\
6.0\end{array}$ & $\begin{array}{l}6 \cdot 0 \\
6 \cdot 2\end{array}$ & $\begin{array}{l}6 \cdot 6 \\
6 \cdot 6\end{array}$ & $\begin{array}{l}6 \cdot 1 \\
6 \cdot 5\end{array}$ & $\begin{array}{l}6 \cdot 7 \\
7 \cdot 0\end{array}$ & $\begin{array}{l}6 \cdot 9 \\
7 \cdot 1\end{array}$ & $\begin{array}{l}7 \cdot 1 \\
6 \cdot 8\end{array}$ & $\begin{array}{l}6.9 \\
7 \cdot 0\end{array}$ & $\begin{array}{l}\text { R. } \\
\text { L. }\end{array}$ & G5 \\
\hline & $\begin{array}{l}6 \cdot 1 \\
6 \cdot 0\end{array}$ & $\begin{array}{l}6 \cdot 5 \\
5 \cdot 6\end{array}$ & $\begin{array}{l}6 \cdot 2 \\
6 \cdot 3\end{array}$ & $\begin{array}{l}6 \cdot 6 \\
6 \cdot 8\end{array}$ & $\begin{array}{l}6 \cdot 7 \\
7 \cdot 0\end{array}$ & $\begin{array}{l}6 \cdot 6 \\
7 \cdot 0\end{array}$ & $\begin{array}{l}7 \cdot 5 \\
6.8\end{array}$ & $\begin{array}{l}6 \cdot 9 \\
6 \cdot 6\end{array}$ & $\begin{array}{l}\mathbf{R} . \\
\mathbf{L} .\end{array}$ & F3 \\
\hline & $\begin{array}{l}5 \cdot 8 \\
5.8\end{array}$ & $\begin{array}{l}6 \cdot 0 \\
6 \cdot 0\end{array}$ & $\begin{array}{l}6 \cdot 7 \\
6 \cdot 4\end{array}$ & $\begin{array}{l}6 \cdot 6 \\
6 \cdot 7\end{array}$ & $\begin{array}{l}5 \cdot 8 \\
6 \cdot 8\end{array}$ & & $\begin{array}{l}7 \cdot 3 \\
7 \cdot 0\end{array}$ & $\begin{array}{l}6 \cdot 8 \\
6.9\end{array}$ & $\begin{array}{l}\mathrm{R} . \\
\mathrm{L} .\end{array}$ & F6 \\
\hline & $\begin{array}{l}6 \cdot 2 \\
5 \cdot 8\end{array}$ & $\begin{array}{l}5 \cdot 5 \\
6.9\end{array}$ & $\begin{array}{l}6 \cdot 2 \\
6 \cdot 9\end{array}$ & $\begin{array}{l}6 \cdot 5 \\
6 \cdot 6\end{array}$ & $\begin{array}{l}6 \cdot 6 \\
6 \cdot 6\end{array}$ & $\begin{array}{l}7 \cdot 3 \\
7 \cdot 2\end{array}$ & $\begin{array}{l}7.2 \\
7 \cdot 0\end{array}$ & & $\begin{array}{l}\text { R. } \\
\text { L. }\end{array}$ & F8 \\
\hline & & & $\begin{array}{l}6 \cdot 0 \\
5 \cdot 8\end{array}$ & $\begin{array}{l}5 \cdot 8 \\
6 \cdot 4\end{array}$ & $\begin{array}{l}6 \cdot 1 \\
6 \cdot 3\end{array}$ & $\begin{array}{l}6 \cdot 6 \\
6 \cdot 6\end{array}$ & $\begin{array}{l}6.9 \\
6.7\end{array}$ & $\begin{array}{l}7 \cdot 2 \\
6 \cdot 8\end{array}$ & $\begin{array}{l}\text { R. } \\
\text { L. }\end{array}$ & E1 \\
\hline & & & $\begin{array}{l}6 \cdot 3 \\
7 \cdot 3\end{array}$ & $\begin{array}{l}6 \cdot 4 \\
6 \cdot 2\end{array}$ & $\begin{array}{l}6 \cdot 6 \\
6 \cdot 9\end{array}$ & $\begin{array}{l}6 \cdot 4 \\
7 \cdot 1\end{array}$ & $\begin{array}{l}6 \cdot 7 \\
6 \cdot 7\end{array}$ & $\begin{array}{l}6 \cdot 7 \\
6 \cdot 9\end{array}$ & $\begin{array}{l}\text { R. } \\
\text { L. }\end{array}$ & E2 \\
\hline & & $\begin{array}{l}5 \cdot 9 \\
5 \cdot 3\end{array}$ & $\begin{array}{l}6 \cdot 1 \\
6 \cdot 5\end{array}$ & $\begin{array}{l}6 \cdot 3 \\
6 \cdot 6\end{array}$ & $\begin{array}{l}6.9 \\
6.6\end{array}$ & $\begin{array}{l}6 \cdot 6 \\
8 \cdot 2\end{array}$ & $\begin{array}{l}6 \cdot 1 \\
6 \cdot 1\end{array}$ & & $\begin{array}{l}\text { R. } \\
\text { L. }\end{array}$ & $\mathrm{Cl}$ \\
\hline & & & $\begin{array}{l}6 \cdot 1 \\
6 \cdot 0\end{array}$ & $\begin{array}{l}7 \cdot 0 \\
6 \cdot 7\end{array}$ & $\begin{array}{l}6 \cdot 3 \\
7 \cdot 3\end{array}$ & $\begin{array}{l}7 \cdot 8 \\
7 \cdot 4\end{array}$ & $\begin{array}{l}7 \cdot 2 \\
6 \cdot 8\end{array}$ & $\begin{array}{l}6.4 \\
6.9\end{array}$ & $\begin{array}{l}\text { R. } \\
\text { L. }\end{array}$ & $\mathrm{C} 2$ \\
\hline $\begin{array}{c}5 \cdot 2 \\
\pm 0.42\end{array}$ & $\begin{array}{c}5.9 \\
\pm 0.31\end{array}$ & $\begin{array}{c}6 \cdot 1 \\
\pm 0.37\end{array}$ & $\begin{array}{c}6.4 \\
\pm 0.40\end{array}$ & $\begin{array}{c}6.6 \\
\pm 0.30\end{array}$ & $\begin{array}{c}6.7 \\
\pm 0.41\end{array}$ & $\begin{array}{c}7 \cdot 0 \\
\pm 0.42\end{array}$ & $\begin{array}{c}6.0 \\
\pm 0.41\end{array}$ & $\begin{array}{c}7 \cdot 0 \\
\pm 0 \cdot 32\end{array}$ & \multicolumn{2}{|c|}{$\begin{array}{l}\text { Mean and } \\
\text { Standard } \\
\text { Deviation }\end{array}$} \\
\hline
\end{tabular}




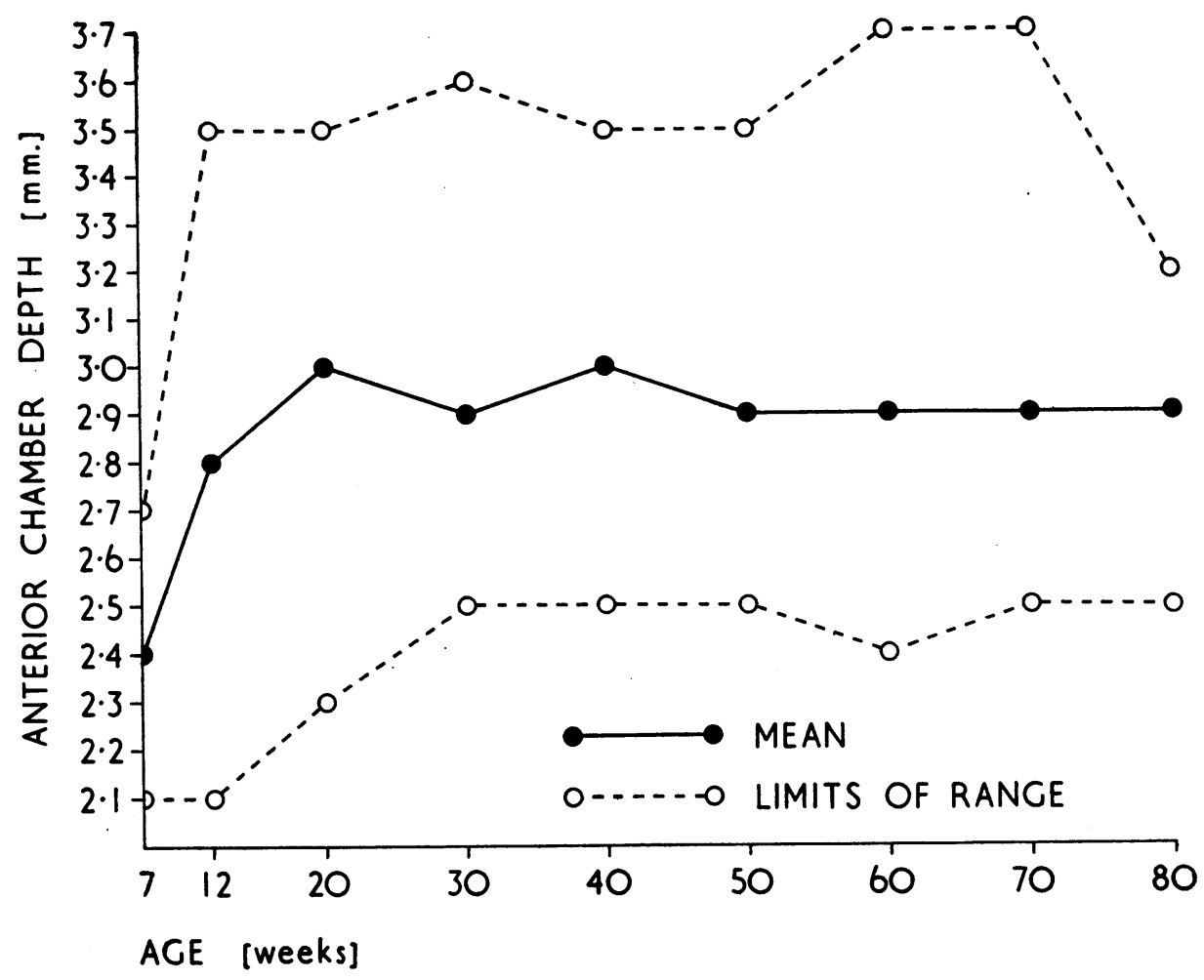

Fig. 1.-Increase in depth of anterior chamber with age.

observations, and over one-third in the first 5 weeks. A flattening in the average radius of curvature of the anterior surface of the lens by $1.8 \mathrm{~mm}$. (from 5.2 to $7.0 \mathrm{~mm}$.) over the period of observation suggests a very considerable change in power. How big the change is depends upon the refractive index of the lens, and there are reasons for believing that in the rabbit this is of the order of 1.60 or more (Sorsby and others, 1961). Taking the value of 1.60 , the corresponding mean front surface powers of the lens at 7 and at 80 weeks are $51 \cdot 3 \mathrm{D}$ and $38 \cdot 1 \mathrm{D}$. This represents a decline in power of $13.2 \mathrm{D}$ (or $11.8 \mathrm{D}$ if the increasing depth of the anterior chamber is taken into account), and compares with $14.8 \mathrm{D}$ for the cornea over the same period (Stone and Leary, 1957). Between 7 and 80 weeks of age the flattening of the anterior surface of the lens is therefore of much the same order as that recorded for the cornea.

It is impossible to apply findings on the rabbit to changes that occur in the human eye during growth, if only because a rabbit of 7 weeks is probably considerably more immature than a human baby at birth. The growth curve of the rabbit eye is in any case likely to be substantially different from that of the human eye. The available evidence is that at full-term birth the 


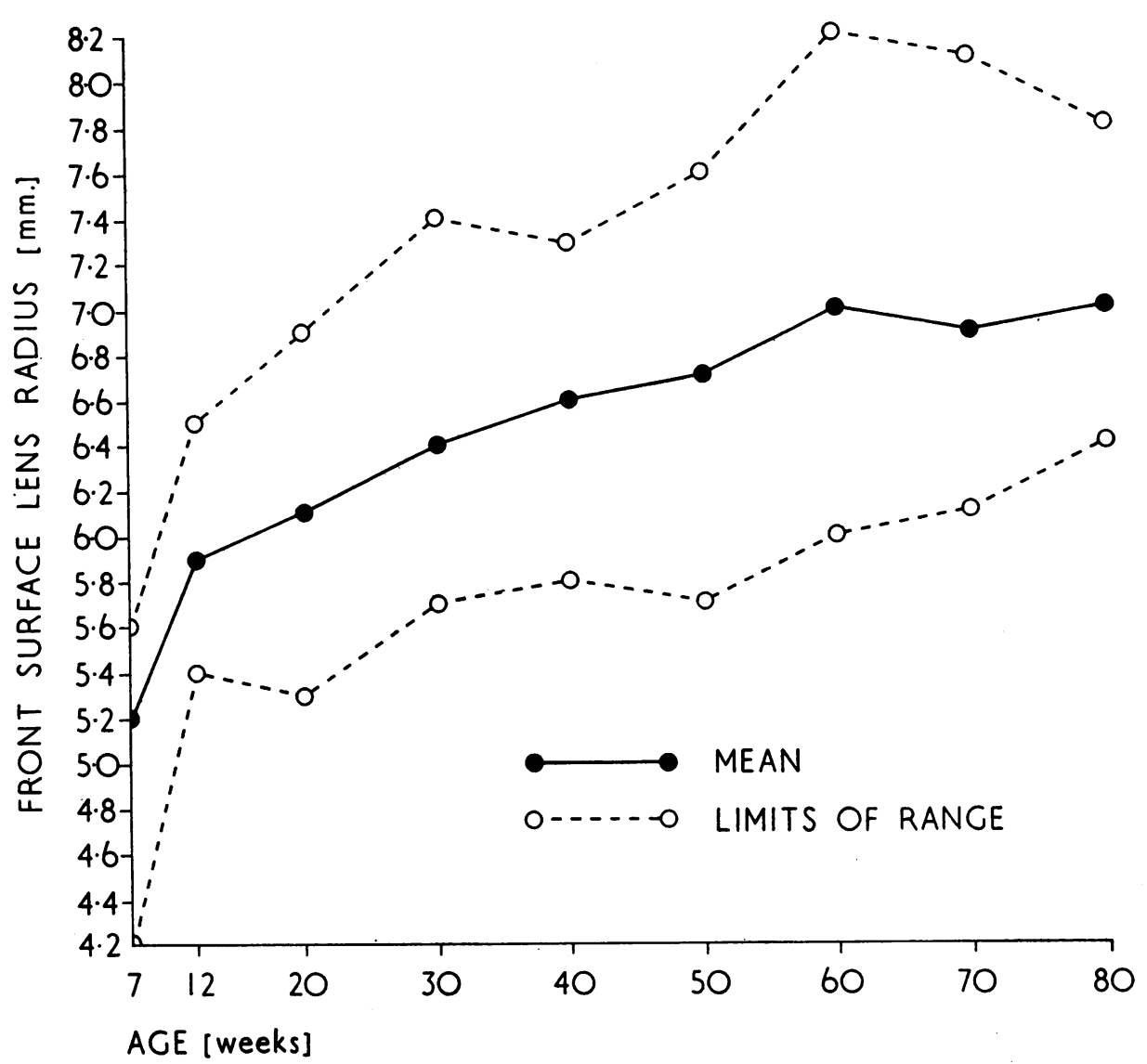

FIG. 2.-Flattening of front surface of lens with age.

human eye is 17.5 to $18.5 \mathrm{~mm}$. long (Sorsby and Sheridan, 1960); this gives an axial elongation of some $6 \mathrm{~mm}$. during post-natal growth. The eyes of newborn rabbits measure about $6 \mathrm{~mm}$., and the average adult value for the rabbit is $18 \mathrm{~mm}$. Both the amount of elongation and the rate of elongation are therefore substantially different from that seen in the human baby, and the compensatory changes are inevitably different too-quantitatively though not necessarily qualitatively.

\section{REFERENCES}

Chou, I. (1954). J. Formosan med. Ass., 53, 483.

Sorsby, A., Benjamin, B., DaVeY, J. B., SHeridan, M., and TANner, J. M. (1957). "Emmetropia and its Aberrations". M.R.C. spec. Rep. Ser. No. 293, H.M.S.O., London. , and SHERIDAN, M. (1961). "Refraction and its Components during The Growth of the Eye after the Age of Three". M.R.C. spec. Rep. Ser. (in the press). and SHERIDAN, M. (1953). Brit. J. Ophthal., 37, 555.

(1960). J. Anat., 94, 192.

Stone, J., and LearY, G. (1957). Brit. J. physiol. Optics, n.s. 14, 90. 